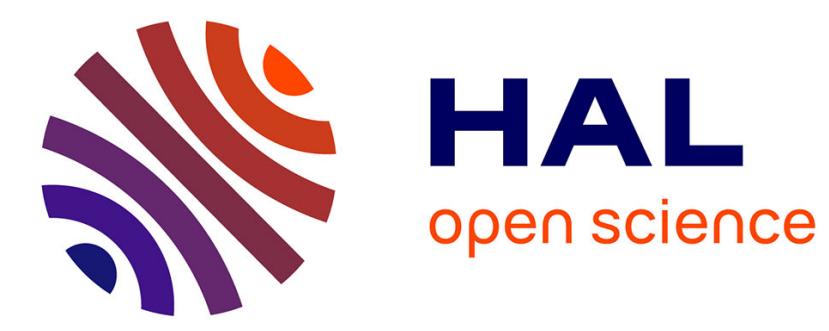

\title{
Surface integral equations for electromagnetic testing: the low-frequency and high-contrast case
} Audrey Vigneron, Édouard Demaldent, Marc Bonnet

\section{To cite this version:}

Audrey Vigneron, Édouard Demaldent, Marc Bonnet. Surface integral equations for electromagnetic testing: the low-frequency and high-contrast case. IEEE Transactions on Magnetics, 2014, 50, pp.7002704. 10.1109/TMAG.2013.2283297 . hal-00937766

\section{HAL Id: hal-00937766 https://hal.inria.fr/hal-00937766}

Submitted on 20 Mar 2014

HAL is a multi-disciplinary open access archive for the deposit and dissemination of scientific research documents, whether they are published or not. The documents may come from teaching and research institutions in France or abroad, or from public or private research centers.
L'archive ouverte pluridisciplinaire HAL, est destinée au dépôt et à la diffusion de documents scientifiques de niveau recherche, publiés ou non, émanant des établissements d'enseignement et de recherche français ou étrangers, des laboratoires publics ou privés. 


\title{
Surface integral equations for electromagnetic testing: the low-frequency and high-contrast case
}

\author{
Audrey Vigneron ${ }^{1}$, Edouard Demaldent ${ }^{1}$, Marc Bonnet ${ }^{2}$ \\ ${ }^{1}$ CEA, LIST, Saclay, 91191 Gif-sur-Yvette, France \\ 2 POEMS (UMR 7231 CNRS-INRIA-ENSTA), ENSTA, 828, boulevard des Maréchaux, 91762 Palaiseau Cedex, France
}

\begin{abstract}
This study concerns boundary element methods applied to electromagnetic testing, for a wide range of frequencies, conductivities and permeabilities. The eddy current (EC) approximation cannot handle all configurations, while numerical instabilities at low frequency or for highly contrasted media affect the Maxwell formulation. We examine on a test example how the performance of several Maxwell and EC formulations is affected by frequency, conductivity and permeability. Among those tested, we propose a weighted loop-tree Maxwell formulation which is found to be the only one yielding satisfactory results in all considered configurations.
\end{abstract}

Index Terms-Eddy currents, low frequency, Maxwell equations, surface integral equations.

\section{INTRODUCTION}

$\mathbf{T}$ HIS study is motivated by the need to efficiently simulate complex configurations of electromagnetic nondestructive testing (ENDT). Complexity may lie in the geometry (non-axisymmetric parts) or in the cohabitation of different models, namely an eddy current model (EC) in conductive parts, a magneto-static model in non-conductive magnetic parts (e.g. ferrite cores) and Maxwell equations in parts where the displacement current cannot be neglected or in weakly conductive parts tested at higher frequencies (e.g. composite media). Here we restrict the study to isotropic and piecewise homogeneous linear media. The boundary element method (BEM) allows intuitive domain decomposition. Moreover, the significant reduction of unknowns compared to domain discretization methods permits the use of a direct solver for most of our configurations.

Due to the difficulty in developing a stable BEM formulation for the wide range of frequencies and physical parameters, practical computations are usually based on BEM formulations that are specific to models or ranges of parameters. For example, the Maxwell formulation Poggio-Miller-ChangHarrington-Wu-Tsai (PMCHWT) with Rao-Wilton-Glisson (RWG) or Rooftop basis functions [1], [2]) suffers from numerical noise at low frequency or in presence of highly contrasted media, a difficulty which can be overcome for dielectric materials [1], [3]. Hence, eddy current (EC) formulations [4] are preferred for highly conductive bodies at low frequencies, although they generally require the introduction of additional (scalar) unknowns. In this paper, we examine on a test example how the performance of several Maxwell and EC formulations, which differ on how the contributions of subdomains are combined or on whether or not the loop-tree decomposition is used, is affected by frequency, permeability and conductivity. The aim of this study is to provide a robust formulation with a good ratio between accuracy and the number of unknowns.

Corresponding author: A. Vigneron (email: audrey.vigneron@cea.fr).

\section{TRANSMISSION PROBLEM: SURFACE INTEGRAL EQUATIONS}

Consider a bounded body $\Omega_{1} \subset \mathbb{R}^{3}$ with its parameters ( $\epsilon_{1}^{\prime}$ the dielectric permittivity, $\mu_{1}$ the permeability, $\sigma$ the conductivity and $\epsilon_{1}=\epsilon_{1}^{\prime}-i \sigma / \omega$ the complex permittivity), outward normal vector $\mathbf{n}$ and the surrounding air filling the complementary domain $\left(\Omega_{0}=\mathbb{R}^{3} \backslash \overline{\Omega_{1}}\right.$ with $\epsilon_{0}$ and $\mu_{0}$ the permittivity and permeability of vacuum).

We can write two tangential and two normal surface integral equations for the tangential and normal components (respectively denoted by $\left.\right|_{\times}$and $\left.\right|_{\mathbf{n}}$ ) of the electric and magnetic fields $\mathbf{E}$ and $\mathbf{H}$ (integrals are taken as Cauchy principal values), [4]:

$$
\begin{gathered}
\left.\mathbf{E}_{0}^{i n c} \delta_{\ell 0}\right|_{\times}=i \omega \mu_{\ell} \mathbf{\Psi}_{V}^{\ell}(\mathbf{J})+\epsilon_{r, \ell}^{-1} \nabla \Psi_{S}^{\ell}\left(\mathbf{E}_{0 n}\right) \\
+\nabla \times \mathbf{\Psi}_{V}^{\ell}(\mathbf{M})-\frac{(-1)^{\delta_{\ell 0}}}{2} \mathbf{n} \times\left.\mathbf{M}\right|_{\times} \\
\left.\mathbf{H}_{0}^{i n c} \delta_{\ell 0}\right|_{\times}=i \omega \epsilon_{\ell} \mathbf{\Psi}_{V}^{\ell}(\mathbf{M})+\mu_{r, \ell}^{-1} \nabla \Psi_{S}^{\ell}\left(\mathbf{H}_{0 n}\right) \\
-\nabla \times \mathbf{\Psi}_{V}^{\ell}(\mathbf{J})+\frac{(-1)^{\delta_{\ell 0}}}{2} \mathbf{n} \times\left.\mathbf{J}\right|_{\times} \\
\left.\mathbf{E}_{0}^{i n c} \delta_{\ell 0}\right|_{\mathbf{n}}=i \omega \mu_{\ell} \mathbf{\Psi}_{V}^{\ell}(\mathbf{J})+\nabla \times \mathbf{\Psi}_{V}^{\ell}(\mathbf{M}) \\
+\epsilon_{r, \ell}^{-1} \nabla \Psi_{S}^{\ell}\left(\mathbf{E}_{0 n}\right)+\left.\frac{(-1)^{\delta_{\ell 0}}}{2 \epsilon_{r, \ell}}\left(\mathbf{E}_{0 n}\right)\right|_{\mathbf{n}} \\
\left.\mathbf{H}_{0}^{i n c} \delta_{\ell 0}\right|_{\mathbf{n}}=i \omega \epsilon_{\ell} \mathbf{\Psi}_{V}^{\ell}(\mathbf{M})-\nabla \times \mathbf{\Psi}_{V}^{\ell}(\mathbf{J}) \\
+\mu_{r, \ell}^{-1} \nabla \Psi_{S}^{\ell}\left(\mathbf{H}_{0 n}\right)+\left.\frac{(-1)^{\delta_{\ell 0}}}{2 \mu_{r, \ell}}\left(\mathbf{H}_{0 n}\right)\right|_{\mathbf{n}},
\end{gathered}
$$

where unknown surface field are defined by $\mathbf{J}:=-\left.\mathbf{H}_{\ell}\right|_{\times}$, $\mathbf{M}:=\left.\mathbf{E}_{\ell}\right|_{\times}, \mathbf{E}_{0 n}:=\left.\epsilon_{r, \ell} \mathbf{E}_{\ell}\right|_{\mathbf{n}}$ and $\mathbf{H}_{0 n}:=\left.\mu_{r, \ell} \mathbf{H}_{\ell}\right|_{\mathbf{n}}$, $\ell \in\{0,1\}$ refers to $\Omega_{\ell}, \delta_{\ell 0}$ is the Kronecker symbol, $\mu_{r, 1}=\mu_{1} / \mu_{0}, \epsilon_{r, 1}=\epsilon_{1} / \epsilon_{0}$ and $\mathbf{E}_{0}^{i n c}, \mathbf{H}_{0}^{i n c}$ are the electric and magnetic incident fields (here obtained by Biot-Savart law).

The scalar and vector single layer potentials $\Psi_{S}^{\ell}, \Psi_{V}^{\ell}$ appearing in (1)-(4) are, respectively, defined by

$$
\begin{aligned}
\Psi_{S}^{\ell}(X)(\mathbf{r}) & :=\int_{\Gamma} X\left(\mathbf{r}^{\prime}\right) g_{\kappa_{\ell}}\left(\mathbf{r}-\mathbf{r}^{\prime}\right) d \mathbf{r}^{\prime}, \\
\Psi_{V}^{\ell}(\mathbf{X})(\mathbf{r}) & :=\int_{\Gamma} \mathbf{X}\left(\mathbf{r}^{\prime}\right) g_{\kappa_{\ell}}\left(\mathbf{r}-\mathbf{r}^{\prime}\right) d \mathbf{r}^{\prime},
\end{aligned}
$$




$$
\left[\begin{array}{cccc}
\alpha_{0} \widetilde{\mathbf{Z}}_{0}^{J J}+\alpha_{1} \widetilde{\mathbf{Z}}_{1}^{J J} & \alpha_{0} \mathbf{Z}_{0}^{J M}+\alpha_{1} \mathbf{Z}_{1}^{J M} & 0 & \alpha_{0} \mathbf{Z}_{0}^{J E}+\alpha_{1} \mathbf{Z}_{1}^{J E} \\
\beta_{0} \mathbf{Z}_{0}^{M J}+\beta_{1} \mathbf{Z}_{1}^{M J} & \beta_{0} \widetilde{\mathbf{Z}}_{0}^{M M}+\beta_{1} \widetilde{\mathbf{Z}}_{1}^{M M} & \beta_{0} \mathbf{Z}_{0}^{M H}+\beta_{1} \mathbf{Z}_{1}^{M H} & 0 \\
a_{0} \mathbf{Z}_{0}^{E J}+a_{1} \mathbf{Z}_{1}^{E J} & a_{0} \mathbf{Z}_{0}^{E M}+a_{1} \mathbf{Z}_{1}^{E M} & 0 & a_{0} \mathbf{Z}_{0}^{E E}+a_{1} \mathbf{Z}_{1}^{E E} \\
b_{0} \mathbf{Z}_{0}^{H J}+b_{1} \mathbf{Z}_{1}^{H J} & b_{0} \mathbf{Z}_{0}^{H M}+b_{1} \mathbf{Z}_{1}^{H M} & b_{0} \mathbf{Z}_{0}^{H H}+b_{1} \mathbf{Z}_{1}^{H H} & 0
\end{array}\right]\left\{\begin{array}{c}
\mathbf{X}^{J} \\
\mathbf{X}^{M} \\
\mathbf{X}^{H} \\
\mathbf{X}^{E}
\end{array}\right\}=\left\{\begin{array}{c}
\alpha_{0} \mathbf{Y}^{J} \\
\beta_{0} \mathbf{Y}^{M} \\
a_{0} \mathbf{Y}^{E} \\
b_{0} \mathbf{Y}^{H}
\end{array}\right\}
$$

where the Green's function is defined as

$$
g_{\kappa_{\ell}}\left(\mathbf{r}-\mathbf{r}^{\prime}\right):=\frac{\exp \left(-i \kappa_{\ell}\left|\mathbf{r}-\mathbf{r}^{\prime}\right|\right)}{4 \pi\left|\mathbf{r}-\mathbf{r}^{\prime}\right|}, \quad \kappa_{\ell}=\omega \sqrt{\epsilon_{\ell} \mu_{\ell}} .
$$

If $\kappa_{\ell} \neq 0$ we have

$$
\begin{aligned}
\epsilon_{r, \ell}^{-1} \nabla \Psi_{S}^{\ell}\left(\mathbf{E}_{0 n}\right) & =\frac{i \omega \mu_{\ell}}{\kappa_{\ell}^{2}} \nabla \nabla \cdot \Psi_{V}^{\ell}(\mathbf{J}), \\
\mu_{r, \ell}^{-1} \nabla \Psi_{S}^{\ell}\left(\mathbf{H}_{0 n}\right) & =\frac{i \omega \epsilon_{\ell}}{\kappa_{\ell}^{2}} \nabla \nabla \cdot \Psi_{V}^{\ell}(\mathbf{M}) .
\end{aligned}
$$

The surface $\Gamma$ can be discretized into triangular or quadrilateral patches. The surface unknowns are approximated by:

$$
\begin{aligned}
\mathbf{J} & =\sum_{b=1}^{N_{\text {edge }}} \mathbf{X}_{b}^{J} \boldsymbol{\varphi}_{b}, \quad \mathbf{M}=\sum_{b=1}^{N_{\text {edge }}} \mathbf{X}_{b}^{M} \boldsymbol{\varphi}_{b}, \\
\mathbf{E}_{0 n} & =\sum_{b=1}^{N_{\text {elt }}} \mathbf{X}_{b}^{E} \varphi_{b}, \quad \mathbf{H}_{0 n}=\sum_{b=1}^{N_{\text {elt }}} \mathbf{X}_{b}^{H} \varphi_{b},
\end{aligned}
$$

where $\varphi_{b}$ is the RWG/Rooftop function associated with the $b$-th edge of the triangular/quadrilateral mesh, $\varphi_{b}$ is the pulse function, equal to 1 in the $b$-th triangle/quadrilateral and 0 elsewhere, and the coefficients $\mathbf{X}^{J / M / E / H}$ are the unknowns of the discretized problem.

After multiplying (1)-(4) respectively by the weighting factors $\alpha_{\ell}, \beta_{\ell}, a_{\ell}, b_{\ell}$ and summing contributions of $\Omega_{0}$ and $\Omega_{1}$ we obtain the system given by (12) where the $\mathbf{Y}$ subvectors and $\mathbf{Z}$ submatrices are defined in Appendix A. We obtain a general eddy current system in the quasistationary approximation case characterized by $\epsilon_{0} \omega / \sigma \ll 1$ and $\operatorname{diam}\left(\Omega_{1}\right) \omega \sqrt{\epsilon_{0} \mu_{0}} \ll 1$, which leads to neglect the dielectric permittivity $\epsilon_{\ell}^{\prime}$ and to set $\kappa_{0}=0$ [5].

When $\kappa_{\ell} \neq 0$, (8) can be substituted into (1) and (9) into (2). Multiplying the modified versions of (1) and (2) by the weighting factors $\alpha_{\ell}$ and $\beta_{\ell}$ and summing contributions of $\Omega_{0}$ and $\Omega_{1}$, we obtain the Maxwell system given (with entries defined in Appendix A) by

$$
\begin{array}{r}
{\left[\begin{array}{cc}
\alpha_{0} \mathbf{Z}_{0}^{J J}+\alpha_{1} \mathbf{Z}_{1}^{J J} & \alpha_{0} \mathbf{Z}_{0}^{J M}+\alpha_{1} \mathbf{Z}_{1}^{J M} \\
\beta_{0} \mathbf{Z}_{0}^{M J}+\beta_{1} \mathbf{Z}_{1}^{M J} & \beta_{0} \mathbf{Z}_{0}^{M M}+\beta_{1} \mathbf{Z}_{1}^{M M}
\end{array}\right]\left\{\begin{array}{c}
\mathbf{X}^{J} \\
\mathbf{X}^{M}
\end{array}\right\}} \\
=\left\{\begin{array}{c}
\alpha_{0} \mathbf{Y}^{J} \\
\beta_{0} \mathbf{Y}^{M}
\end{array}\right\}
\end{array}
$$

A Helmholtz decomposition (loop-tree basis functions) can be applied to (13). The resulting system has the form

$$
\widehat{\mathbf{Z}} \widehat{\mathbf{X}}=\widehat{\mathbf{Y}}
$$

with $\widehat{\mathbf{Z}}:=\mathbf{P Z} \mathbf{P}^{*}, \widehat{\mathbf{X}}:=\left(\mathbf{P}^{*}\right)^{-1} \mathbf{X}, \widehat{\mathbf{Y}}:=\mathbf{P Y}$ and where $\mathbf{P}$ effects the change to loop-tree basis functions. In partitioned form, system (14) has the form

$$
\widehat{\mathbf{Z}}^{a b}=\left[\begin{array}{ll}
\mathbf{Z}_{L L}^{a b} & \mathbf{Z}_{L T}^{a b} \\
\mathbf{Z}_{T L}^{a b} & \mathbf{Z}_{T T}^{a b}
\end{array}\right], \quad \widehat{\mathbf{X}}^{b}=\left\{\begin{array}{c}
\mathbf{X}_{L}^{b} \\
\mathbf{X}_{T}^{b}
\end{array}\right\}, \quad \widehat{\mathbf{Y}}^{a}=\left\{\begin{array}{c}
\mathbf{Y}_{L}^{a} \\
\mathbf{Y}_{T}^{a}
\end{array}\right\},
$$

where $(a, b)=(J, M)$ while subscripts $L$ and $T$ refer to loop and tree functions.

\section{INTEGRAL FORMULATIONS: A COMPARATIVE STUDY}

The defining characteristics of the formulations presented in this section are summarized in Table I.

The PMCHWT formulation, widely used for dielectric or conductive bodies, suffers from low-frequency breakdown. The loop-tree decomposition (14) is a popular way to overcome this low-frequency breakdown for dielectric bodies modelled using triangular patches. In the low-frequency limit, the first and second terms of $\mathbf{Z}^{J J}$ and $\mathbf{Z}^{M M}$ in (13) respectively behave like $O(\omega)$ and $O\left(\omega^{-1}\right)$, causing numerical noise. In (14) the second term of $\mathbf{Z}^{J J}$ and $\mathbf{Z}^{M M}$ vanishes when applied to, or tested with, loop basis functions. Here, we directly adapt to rectangular patches the matrix $\mathbf{P}$ proposed in [1] for triangular patches and we apply the decomposition to conductive bodies. We will name this formulation LT.

The EC formulation introduced in [6], called here LTEC, is retrieved from (14) by suppressing the tree terms of the electric current density $\mathbf{J}_{T}$ then imposing $\epsilon_{\ell}^{\prime}=0$.

Note that $\alpha_{0}=\alpha_{1}$ and $\beta_{0}=\beta_{1}$ for all these formulations, causing the terms $\mathbf{I}_{\times}$arising in $\mathbf{Z}^{J M}$ and $\mathbf{Z}^{M J}$ to cancel out. In addition, other formulations for which this cancellation does not occur are also available, such as the Müller formulation used for high contrasted dielectric bodies. The corresponding matrix is however not diagonally dominant, which can make the set of RWG basis functions unsuitable as test functions. An alternative version (N-Müller [3]) consists in testing (13) with $\mathbf{n} \times($ RWG/Rooftop) basis functions.

Still other formulations are derived from the system (12) by setting $\epsilon_{\ell}^{\prime}=0$. The EC1 and EC2 formulations of [4] involve three and four unknowns, respectively (whereas Maxwell formulations involve just two) and also do not experience $\mathbf{I}_{\times}$ cancellations. Suitable testing functions for EC1 and EC2 are $\mathbf{n} \times($ RWG/Rooftop) basis functions.

Formulations EC1 and EC2 use equations written for one medium only (either $\ell=0$ or $\ell=1$ ), whereas all other mentioned formulations use weighted combinations of equations arising for each medium. This prompts us to consider variants of the PMCHWT and LT formulations, respectively denoted by PMCHWTw and LTw, which are also based on single-medium equations. 
TABLE I

FORMULATIONS

\begin{tabular}{|c|c|c|c|c|c|}
\hline Label & System & Coefficient & Test function & Basis function & $\epsilon_{\ell}^{\prime}=0$ \\
\hline PMCHWT & (13) & $\alpha_{0 / 1}=\beta_{0 / 1}=1$ & Rooftop/RWG & Rooftop/RWG & no \\
\hline LT & (14) & $\alpha_{0 / 1}=\beta_{0 / 1}=1$ & Rooftop/RWG & Rooftop/RWG & no \\
\hline LTEC & (14) & $\alpha_{0 / 1}=\beta_{0 / 1}=1$ & Rooftop/RWG & Rooftop/RWG & yes \\
\hline Müller & (13) & $\alpha_{0}=-\epsilon_{0}, \alpha_{1}=\epsilon_{1}, \beta_{0}=\mu_{0}, \beta_{1}=-\mu_{1}$ & Rooftop/RWG & Rooftop/RWG & no \\
\hline N-Müller & (13) & $\alpha_{0}=-\epsilon_{0}, \alpha_{1}=\epsilon_{1}, \beta_{0}=\mu_{0}, \beta_{1}=-\mu_{1}$ & $\mathbf{n} \times($ Rooftop/RWG $)$ & Rooftop/RWG & no \\
\hline EC1 & (12) & $\begin{array}{l}\alpha_{0}=\beta_{1}=0, \alpha_{1}=\beta_{0}=1 \\
a_{0 / 1}=b_{0}=0, b_{1}=1\end{array}$ & $\begin{array}{l}\mathbf{n} \times(\text { Rooftop/RWG }) \\
\text { pulse }\end{array}$ & $\begin{array}{l}\text { Rooftop/RWG } \\
\text { pulse }\end{array}$ & yes \\
\hline EC2 & (12) & $\begin{array}{l}\alpha_{1}=\beta_{0}=0, \alpha_{0}=\beta_{1}=1 \\
a_{1}=b_{1}=0, a_{0}=b_{0}=1\end{array}$ & $\begin{array}{l}\mathbf{n} \times(\text { Rooftop/RWG }) \\
\text { pulse }\end{array}$ & $\begin{array}{l}\text { Rooftop/RWG } \\
\text { pulse }\end{array}$ & yes \\
\hline PMCHWTw & (13) & $\alpha_{0}=\beta_{1}=0, \alpha_{1}=\beta_{0}=1$ & Rooftop/RWG & Rooftop/RWG & no \\
\hline LTw & (14) & $\alpha_{1}=\beta_{0}=0, \alpha_{0}=\beta_{1}=1$ & Rooftop/RWG & Rooftop/RWG & no \\
\hline
\end{tabular}

\section{NUMERICAL RESUlTS}

The above formulations are now examined on a configuration representative of ENDT experiments, hence involving a conductive body, is considered. We compare the variation of impedance $(\Delta Z=\Delta R+i \Delta X)$ of a coil $\left(r_{i n t}=1 \mathrm{~mm}\right.$, $\left.r_{\text {ext }}=2 \mathrm{~mm}, h=1 \mathrm{~mm}, I=1 \mathrm{~A}, 1 \mathrm{turn}\right)$ placed $0.1 \mathrm{~mm}$ above a conductive cylindrical plate $(R=5 \mathrm{~mm}, H=1 \mathrm{~mm})$ for the formulations of table I. The plate surface is discretized by 3738 quadrilateral patches (similar results, not shown for brevity, have also been obtained using triangular patches). The reference result is computed by axisymmetric finite integration technique whose computation domain is chosen sufficiently large $(50 \mathrm{~mm} \times 50 \mathrm{~mm})$ to neglect the edge effects.

For the case of a non-magnetic plate $\left(\mu_{r, 1}=1\right)$ we vary the frequency $(f=\omega / 2 \pi)$ from $100 \mathrm{~Hz}$ to $100 \mathrm{MHz}$ for a skin depth $\delta=1.59 \mathrm{~mm}$ (with $\left.\delta=\sqrt{2 /\left(\omega \mu_{1} \sigma_{1}\right)}\right)$. Relative discrepancies (with respect to reference values) on the evaluation of the impedance variation are shown in Figure 1. Accurate results have been obtained using the LT, LTw and LTEC formulations, while the EC1 formulation is less satisfactory. Moreover, the PMCHWT and PMCHWTw formulations seem to suffer from low-frequency breakdown for $f<10 \mathrm{kHz}$ and $f<100 \mathrm{kHz}$, respectively. Results for EC2, Müller and NMüller were found to be unsatisfactory and are not presented. In Figure 2, the effect of the skin depth is investigated (with
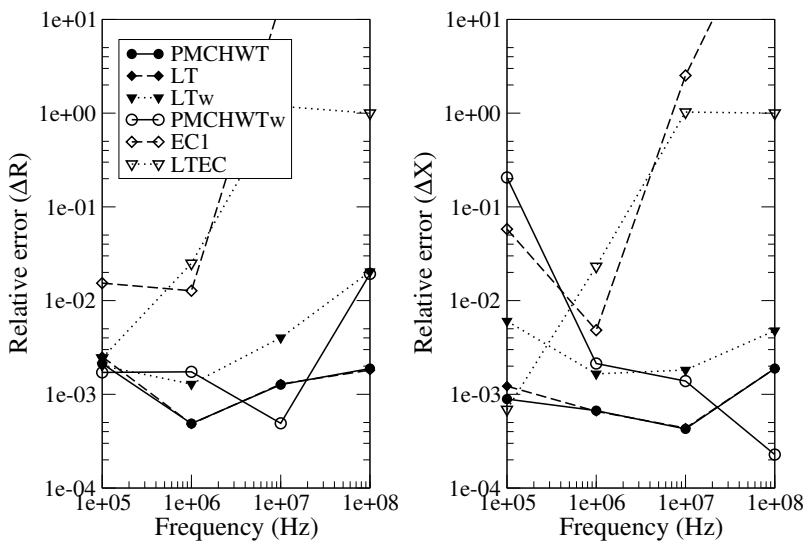

Fig. 2. Relative error on impedance variation for $\sigma_{1}=1 \mathrm{MS} / \mathrm{m}, \mu_{r, 1}=1$.

$1.59 \mathrm{~mm} \geq \delta \geq 50.3 \mu \mathrm{m}$ and $\sigma_{1}$ set to $\left.1 \mathrm{MS} / \mathrm{m}\right)$. Formulations EC1 and LTEC diverge quickly. Accurate results have been obtained using the LT, LTw and PMCHWT formulations.

For the case of a magnetic plate $\left(\mu_{r, 1}=100\right)$, results are shown only for these three formulations, the others having yielded erroneous results. We vary the frequency from $1 \mathrm{~Hz}$ to $1 \mathrm{MHz}$, with the skin depth set to $\delta=1.59 \mathrm{~mm}$. The results of Figure 3 indicate that LTw is more accurate than LT and PMCHWT, while PMCHWT suffers from low-frequency
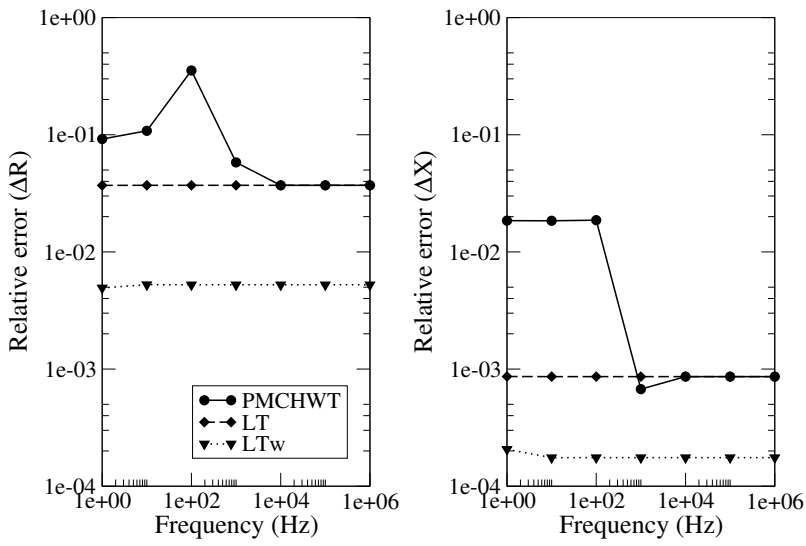

Fig. 3. Relative error on impedance variation for $\delta=1.59 \mathrm{~mm}, \mu_{r, 1}=100$
Fig. 1. Relative error on impedance variation for $\delta=1.59 \mathrm{~mm}, \mu_{r, 1}=1$.
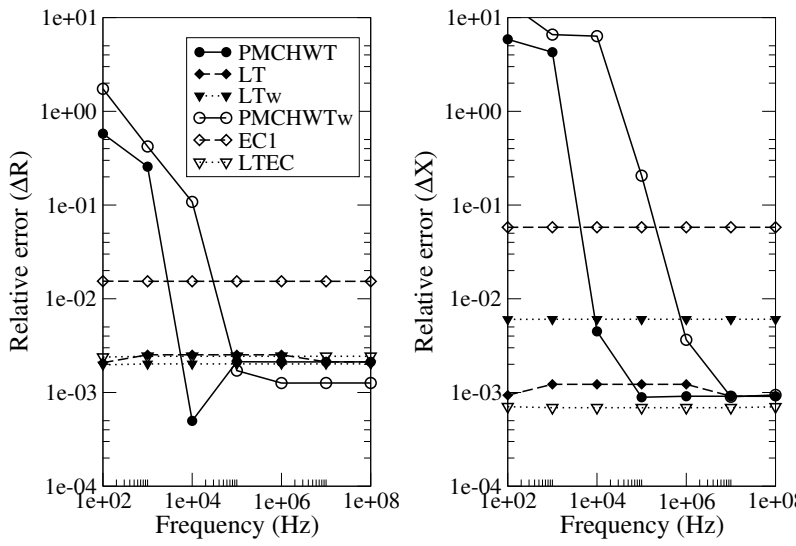

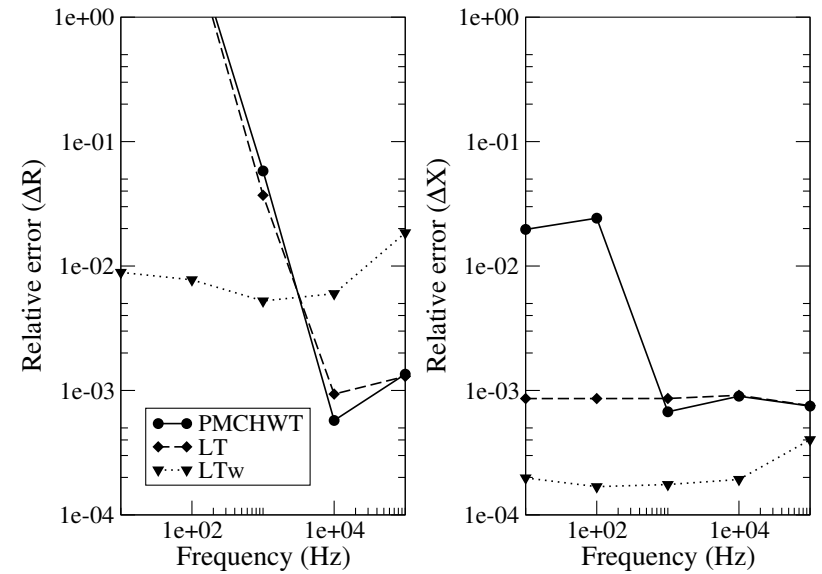

Fig. 4. Relative error on impedance variation for $\sigma_{1}=1 \mathrm{MS} / \mathrm{m}, \mu_{r, 1}=100$

breakdown for $f<1 \mathrm{kHz}$. The effect of the skin depth (with $15.9 \mathrm{~mm} \geq \delta \geq 159 \mu \mathrm{m}$ and $\sigma_{1}$ set to $1 \mathrm{MS}$ ) is again investigated in Figure 4. It appears that only the LTw formulation remains effective.

These results are obtained with a Matlab code that is not optimized yet. For each computation, CPU time is mainly dedicated to the matrix fill time and does not exceed 15 minutes if there is enough RAM. It was not our case with EC formulations $(4 \times 3738$ unknowns for Maxwell formulations, $5 \times 3738$ for EC1 and $6 \times 3738$ for EC2).

\section{CONClusion}

In this paper different formulations have been compared for a range of frequency, conductivity and permeability typical of ENDT experiments. The weighted loop-tree Maxwell formulation LTw is the only one yielding satisfactory results for all tried configurations. In a future work, we will study the dependence on physical parameters of the integral operators involved in the LTw formulation, to improve its weighting factors and therefore its performance and stability. Then, we will investigate more complex ENDT configurations and crack modelling.

\section{APPENDIX A}

The various entries in (13) are given by

$$
\begin{aligned}
\mathbf{Z}_{\ell}^{J J} & =i \omega \mu_{\ell}\left(\mathbf{A}_{\times}^{\ell}+\mathbf{C}_{\times}^{\ell}\right), & \mathbf{Z}_{\ell}^{J M} & =\mathbf{B}_{\times}^{\ell}+\frac{(-1)^{\delta_{\ell 0}}}{2} \mathbf{I}_{\times}, \\
\mathbf{Z}_{\ell}^{M J} & =-\mathbf{B}_{\times}^{\ell}-\frac{(-1)^{\delta_{\ell 0}}}{2} \mathbf{I}_{\times}, & \mathbf{Z}_{\ell}^{M M} & =i \omega \epsilon_{\ell}\left(\mathbf{A}_{\times}^{\ell}+\mathbf{C}_{\times}^{\ell}\right), \\
\mathbf{Y}^{J} & =\left\langle\boldsymbol{\varphi}_{t}, \mathbf{E}_{0}^{i n c}\right\rangle_{\times}, & \mathbf{Y}^{M} & =\left\langle\boldsymbol{\varphi}_{t}, \mathbf{H}_{0}^{i n c}\right\rangle_{\times},
\end{aligned}
$$

while additional entries of (12) are given by

$$
\begin{gathered}
\widetilde{\mathbf{Z}}_{\ell}^{J J}=i \omega \mu_{\ell} \mathbf{A}_{\times}^{\ell}, \quad \mathbf{Z}_{\ell}^{J E}=\epsilon_{r, \ell} \mathbf{D}_{\times}^{\ell}, \quad \widetilde{\mathbf{Z}}_{\ell}^{M M}=i \omega \epsilon_{\ell} \mathbf{A}_{\times}^{\ell}, \\
\mathbf{Z}_{\ell}^{M H}=\mu_{r, \ell} \mathbf{D}_{\times}^{\ell}, \quad \mathbf{Z}_{\ell}^{E J}=i \omega \mu_{\ell} \mathbf{A}_{\mathbf{n}}^{\ell}, \quad \mathbf{Z}_{\ell}^{E M}=\mathbf{B}_{\mathbf{n}}^{\ell}, \\
\mathbf{Z}_{\ell}^{H J}=-\mathbf{B}_{\mathbf{n}}^{\ell}, \quad \mathbf{Z}_{\ell}^{H M}=i \omega \epsilon_{\ell} \mathbf{A}_{\mathbf{n}}^{\ell}, \\
\mathbf{Z}_{\ell}^{E E}=\epsilon_{r, \ell}\left(\mathbf{D}_{\mathbf{n}}^{\ell}-\frac{(-1)^{\delta_{\ell 0}}}{2} \mathbf{I}_{\mathbf{n}}\right), \\
\mathbf{Z}_{\ell}^{H H}=\mu_{r, \ell}\left(\mathbf{D}_{\mathbf{n}}^{\ell}-\frac{(-1)^{\delta_{\ell 0}}}{2} \mathbf{I}_{\mathbf{n}}\right),
\end{gathered}
$$

$$
\mathbf{Y}^{E}=\left\langle\varphi_{t}, \mathbf{E}_{0}^{i n c}\right\rangle_{\mathbf{n}}, \quad \mathbf{Y}^{H}=\left\langle\varphi_{t}, \mathbf{H}_{0}^{i n c}\right\rangle_{\mathbf{n}} .
$$

All the above quantities involve the bilinear forms

$$
\begin{array}{rlrl}
\mathbf{A}_{\times}^{\ell} & =\left\langle\boldsymbol{\varphi}_{t}, \mathbf{\Psi}_{V}^{\ell}\left(\boldsymbol{\varphi}_{b}\right)\right\rangle_{\times}, & & \mathbf{A}_{\mathbf{n}}^{\ell}=\left\langle\varphi_{t}, \boldsymbol{\Psi}_{V}^{\ell}\left(\boldsymbol{\varphi}_{b}\right)\right\rangle_{\mathbf{n}}, \\
\mathbf{B}_{\times}^{\ell} & =\left\langle\boldsymbol{\varphi}_{t}, \nabla \times \mathbf{\Psi}_{V}^{\ell}\left(\boldsymbol{\varphi}_{b}\right)\right\rangle_{\times}, & \mathbf{B}_{\mathbf{n}}^{\ell}=\left\langle\varphi_{t}, \nabla \times \mathbf{\Psi}_{V}^{\ell}\left(\boldsymbol{\varphi}_{b}\right)\right\rangle_{\mathbf{n}}, \\
\mathbf{D}_{\times}^{\ell} & =\left\langle\boldsymbol{\varphi}_{t}, \nabla \Psi_{S}^{\ell}\left(\varphi_{b}\right)\right\rangle_{\times}, & \mathbf{D}_{\mathbf{n}}^{\ell}=\left\langle\varphi_{t}, \nabla \Psi_{S}^{\ell}\left(\varphi_{b}\right)\right\rangle_{\mathbf{n}}, \\
\mathbf{I}_{\times}^{\ell} & =\left\langle\boldsymbol{\varphi}_{t}, \mathbf{n} \times \boldsymbol{\varphi}_{b}\right\rangle_{\times}, & \mathbf{I}_{\mathbf{n}}^{\ell}=\left\langle\varphi_{t}, \varphi_{b}\right\rangle_{\mathbf{n}}, \\
\mathbf{C}_{\times}^{\ell} & =\left\langle\boldsymbol{\varphi}_{t}, \frac{1}{\kappa_{\ell}^{2}} \nabla \nabla \cdot \mathbf{\Psi}_{V}^{\ell}\left(\boldsymbol{\varphi}_{b}\right)\right\rangle_{\times}, &
\end{array}
$$

in which the inner products $\langle\cdot, \cdot\rangle_{\times}$and $\langle\cdot, \cdot\rangle_{\mathbf{n}}$ are defined by

$$
\begin{aligned}
\left\langle\mathbf{X}_{1}, \mathbf{X}_{2}\right\rangle_{\times} & =\int_{\Gamma} \mathbf{X}_{1} \cdot\left(\mathbf{n} \times\left(\mathbf{X}_{2} \times \mathbf{n}\right)\right) d \Gamma, \\
\left\langle X_{1}, X_{2}\right\rangle_{\mathbf{n}} & =\int_{\Gamma} X_{1}\left(\mathbf{n} \cdot X_{2}\right) d \Gamma .
\end{aligned}
$$

\section{REFERENCES}

[1] S. Y. Chen, W. C. Chew, J. M. Song, and J.-S. Zhao, "Analysis of low frequency scattering from penetrable scatterers," Geoscience and Remote Sensing, IEEE Transactions on, vol. 39, no. 4, pp. 726-735, 2001.

[2] A. Glisson and D. Wilton, "Simple and efficient numerical methods for problems of electromagnetic radiation and scattering from surfaces," Antennas and Propagation, IEEE Transactions on, vol. 28, no. 5, pp. 593-603, 1980

[3] P. Yla-Oijala and M. Taskinen, "Well-conditioned müller formulation for electromagnetic scattering by dielectric objects," Antennas and Propagation, IEEE Transactions on, vol. 53, no. 10, pp. 3316 - 3323, 2005.

[4] W. M. Rucker, R. Hoschek, and K. R. Richter, "Various bem formulations for calculating eddy currents in terms of field variables," Magnetics, IEEE Transactions on, vol. 31, no. 3, pp. 1336-1341, 1995.

[5] A. Buffa, H. Ammari, and J. Nédélec, "A justification of eddy currents model for the maxwell equations," SIAM Journal on Applied Mathematics, vol. 60 , no. 5, pp. $1805-183,2000$.

[6] R. Hiptmair and J. Ostrowski, "Coupled boundary-element scheme for eddy-current computation," Journal of engineering mathematics, vol. 51, no. 3, pp. 231-250, 2005.

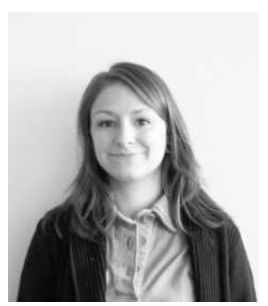

Audrey Vigneron was born in Angers, France, in 1987. She received the M.S. degree in applied mathematics from the University of Rennes 1, Rennes, in 2011. She is currently working toward the Ph.D. degree at the École Polytechnique, at CEA (Commissariat l'Énergie Atomique, France). Her research interests include computational electromagnetics and integral equations.

Edouard Demaldent was born in Paris, France, in 1981. He received the $\mathrm{Ph} . \mathrm{D}$. degree in applied mathematics from the University Paris-IX in 2009 for his research at ONERA (Office National d'Études et de Recherches Aérospatial, France) on computational electromagnetics. Since then, he is working on the modelisation of ENDT configurations, at CEA (Commissariat l'Énergie Atomique, France). His research interests include computational electromagnetics, integral equations and high-order methods.

Marc Bonnet was born in Saverne, France, in 1960. He received his engineer (1983) and doctor (1986) degrees from Ecole Nationale des Ponts et Chaussées (Paris, France) and later (1995) obtained his habilitation from Paris 6 university. He is since 2000 a senior scientist with the French National Center for Scientific Research (CNRS). He is currently based at the Applied Mathematics department of ENSTA (Palaiseau, France). His research interests include integral equation methods for wave propagation problems, and inverse problems. 